Article

\title{
Nanoimprint Resist Material Containing Ultraviolet Reactive Fluorine Surfactant for Defect Reduction in Lithographic Fabrication
}

\author{
Satoshi Takei $^{1,2, *}$ and Atsushi Sekiguchi ${ }^{1}$ \\ 1 Department of Mechanical Systems Engineering, Toyama Prefectural University, Imizu, Toyama \\ 939-0398, Japan \\ 2 Department of Materials Engineering Science, Osaka University, Toyonaka, Osaka 560-8531, Japan \\ * Author to whom correspondence should be addressed; E-Mail: takeis@pu-toyama.ac.jp; \\ Tel.: +81-766-56-7500; Fax: +81-766-56-6131.
}

Received: 9 December 2011; in revised form: 4 January 2012 / Accepted: 5 January 2012 /

Published: 16 January 2012

\begin{abstract}
The generated resist based defects on the template in addition to the presence of particles and contaminants is critical for ultraviolet curing of nanoimprint lithographic fabrication. This procedure is proven to be suitable for advanced resist material design under the process conditions. Nanoimprint resist material containing an ultraviolet reactive fluorine surfactant was developed to modify the fundamental surface interactions between resists and the template for defect reduction in nanoimprint patterning replication. The developed acrylate type nanoimprint resist material containing $4,4,5,5,6,6,7,7,8,8,9,9,10,10,11,11,11$-heptadecafluoro-2-hydroxyundecyl acrylate as an ultraviolet reactive fluorine surfactant, indicated excellent patterning dimensional accuracy by minimizing surface free energy, and having the effect of improving the generated resist based defect numbers on the template, with a $500 \mathrm{~nm}$ contact hole and $2 \mu \mathrm{m}$ line patterns, in the replication of 20 nanoimprint process cycles. This desirable concept using an ultraviolet reactive fluorine surfactant with an acrylate group in the acrylate type nanoimprint resist material is one of the most promising processes ready to be incorporated into mass fabrication in the next generation of electronic devices.
\end{abstract}

Keywords: ultraviolet reactive fluorine surfactant; nanoimprint lithography; resist; defect reduction; surface free energy 


\section{Introduction}

Heading towards mass fabrication for the next nano electronic devices, ultraviolet (UV) curing nanoimprint lithography is one option for the pattern generation of electronic circuits on wafers. In UV curing nanoimprint lithography, the mold of quartz or polymer is used as the template. The fluorinated alkyl silane as a fluorinated self assembled monolayer was coated on the template with one of the surface modification techniques in UV curing nanoimprint lithography. UV curing nanoimprint resist materials are dispensed as droplets on underlayer materials, and then are set with the template with low pressure. The nanoimprint resist materials fill the template and are then solidified by brief UV irradiation, forming the desired pattern. These processes are repeated across the wafer, similar to the step and repeat lithography process.

UV curing nanoimprint lithography is a promising method recently used for next generation device manufacturing because it is of low production cost, high throughput and can be carried out at room temperature. In addition, UV curing nanoimprint lithography takes advantage of the synergetic effects of micro- to nano-sized patterns on various flexible substrates, three-dimensional patterns, direct patterns of metal, and develop-less patterning applications such as magnetic memory structures, organic light-emitting diodes, optical sensor devices, hybrid-laser system, biosensor devices, and biomedical nanospectrometer devices.

UV curing nanoimprint lithography offers the opportunity to relax the very complex mask patterns by avoiding much of the sub-resolution assist features required for ArF lithography at a comparable design rule. In addition, in order to solve the cost issues using mass fabrication methods nanoimprint lithography was investigated in nanoelectronic devices [1]. In advanced electronic device applications using nanoimprint lithography, a UV curing glucose-based resist material from biomass was studied for environmental applicability with $65 \mathrm{~nm}$ dense line patterning [2]. A UV sensitive hard mask underlayer material with terminal methacrylate groups has been developed, in order to improve the generated resist peeling and contamination problem, when the template was removed from the resist after UV irradiation [3]. The formulation of advanced resist materials design of fluorinated silicon-based resist materials in the step and flash nano imprint lithography for the next generation was reported [4]. The thermal nanoimprint lithography for $\mathrm{Au}$ electrodeposition was demonstrated [5]. The shrinkage behaviors and elimination time of bubbles for different cavity sizes and resist thicknesses were investigated [6]. The direct patterning of low-dielectric constant (low-k) materials via nanoimprint lithography was evaluated [7].

However, the UV curing nanoimprint lithography in the mass fabrication of the next generation device manufacturing, faces a challenge because of the various kinds of defects, particles, and contaminants positioned between the nanoimprint resist material and the template Examples of which are resist peeling, dislocations, voids, and impurities. The defect inspections which focused on two key defect types of random non-fill defects occurring during the nanoimprint resist filling process together with repeater defects caused by interactions with particles on the substrate, were summarized [8]. The new proposed nanoimprint resist material design and the nano imprinting processing conditions were investigated to decrease the resist based defect number on the template, when the template was removed from the resist material after pressing the template into the resist 
material [9]. Figure 1 shows the classic optical images of a dirty-template causing the generated resist based defects on the template in the repeatability of nanoimprint process cycles.

Figure 1. Optical images of the generated resist based defects on the template in nanoimprint lithography.
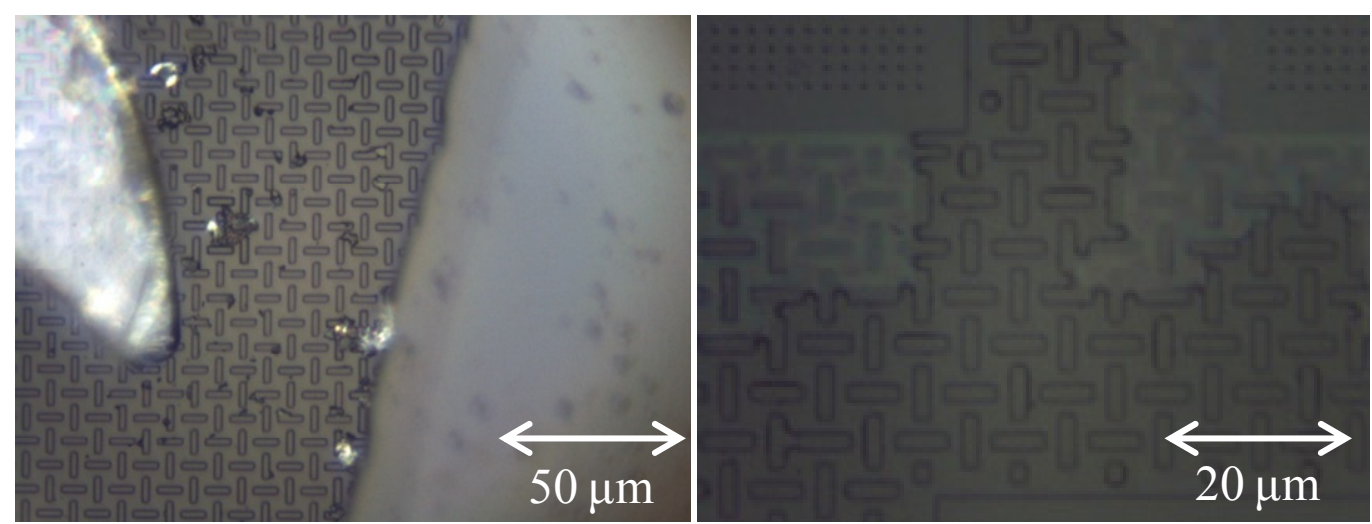

UV curing nanoimprint lithography requires the clean separation of a template from a nanoimprint resist material, and the force required to create this separation must be minimized to decrease the generated resist based defect number on the template. This problem of nanoimprint resist material on the template is critical for mass fabrication using UV curing nanoimprint lithography, when the template is removed from the nanoimprint resist material after pressing the template into the nanoimprint resist material.

Therefore, the nanoimprint resist material containing a UV reactive fluorine surfactant was investigated to modify the fundamental surface interactions between nanoimprint resist material and template for defect reduction in nanoimprint patterning dimensional accuracy, by replication of imprint process cycles. The effect of reducing the defect numbers on the template with a $500 \mathrm{~nm}$ contact hole and $2 \mu \mathrm{m}$ line patterns in the replication of nanoimprint process cycles was demonstrated and is discussed in terms of minimizing the surface free energy of nanoimprint resist material.

\section{Experimental Procedure}

\subsection{UV Curing Nanoimprint Lithography}

It is essentially a stamping process, the nanoimprint lithography mechanically deforms a nanoimprint medium using a three-dimensional template with the desired pattern. The nanoimprint medium is then hardened to produce a negative of the template pattern. UV curing nanoimprint lithography was focused on in this study as the next generation process of choice for the mass fabrication of next generation device manufacturing. Figure 2 shows a schematic of the UV curing nanoimprint lithographic process cycles. The cycles were as follows: (1) The underlayer material TPU-UL (Toyama Prefectural University) is spin-coated on a substrate [9], and then the underlayer-coated substrate is prebaked at $200{ }^{\circ} \mathrm{C}$ for $2 \mathrm{~min}$. The nanoimprint resist material containing a UV reactive fluorine surfactant was dispensed onto the underlayer-coated substrate. Total volume of nanoimprint resist material was $0.15 \mathrm{~mL}$; (2) The template was pressed onto this nanoimprint resist material. The nanoimprint resist material filled thetemplate. To examine the effect of improving the issue of the 
generated resist based defects on thetemplate in the replication of nanoimprint process cycles, the transparent quartz template with a $500 \mathrm{~nm}$ contact hole and $2 \mu \mathrm{m}$ line patterns was prepared. The imprinting force was $17.4 \mathrm{~N}$, and the template was pressed for $2 \mathrm{~min}$; (3) Once the nanoimprint resist material completely filled the template patterns, it was exposed to UV irradiation through the template, activating a UV curing reaction to solidify the molded nanoimprint resist material. The UV irradiation was performed at an energy density of $3.48 \mathrm{~J} / \mathrm{cm}^{2}$; (4) Finally, the template was separated from the polymerized nanoimprint resist material. The cycles were repeated across the wafer, similar to the step-and-repeat lithography processes. The UV curing nanoimprint lithography is repeatable, and the durable template is necessary. This is because the generated resist based defects limit the effectiveness of pattern transfer in nanoimprint process cycles. The template was cleaned before each test of the different nanoimprint resist material, and the optical microscope images on the template were examined every time.

Figure 2. Schematic of process cycles in UV curing nanoimprint lithography: (1) deposition of resist droplets; (2) spreading of resist by nanoimprinting; (3) UV irradiation; and (4) template release.

(1)

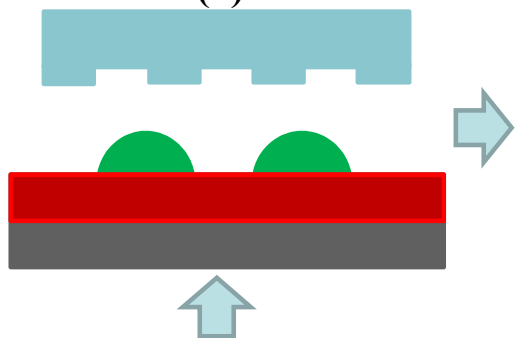

(4)

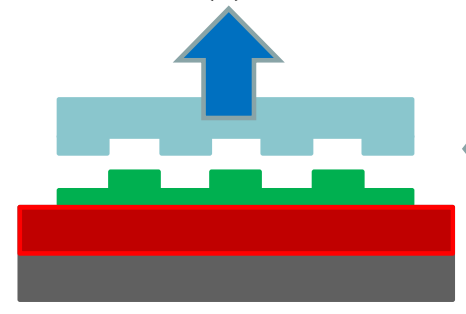

(2)

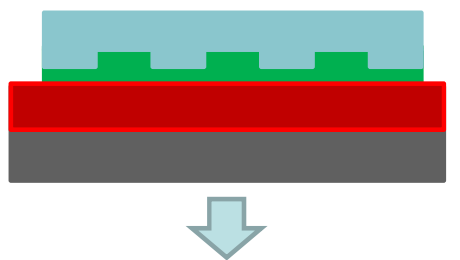

(3)

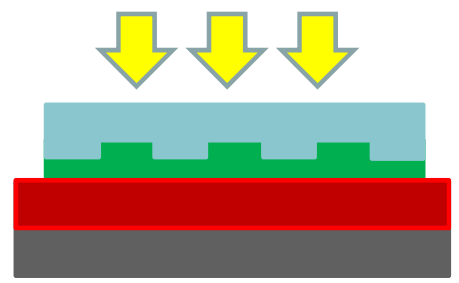

In order to obtain a better understanding of defect reduction on the template in nanoimprint patterning replication, the effect of an ultraviolet reactive fluorine surfactant, reacted with the main components in nanoimprint resist material, was evaluated using the above process cycles of UV curing nanoimprint lithography in this study.

\subsection{Nanoimprint Resist Material Containing a UV Reactive Fluorine Surfactant}

The properties of nanoimprint resist material include low viscosity, low vapor pressure, high sensitivity in UV irradiation, minimal film volumetric shrinkage during UV curing process, suitable mechanical strength, and suitable thermal stability. The low viscosity of the nanoimprint resist material is necessary to enable the dispense technique and the low pressure capillary fill process in UV curing nanoimprint lithography. The low vapor pressure of the nanoimprint resist material allows a smaller droplet size to be used in the dispense process, which increases the drop pattern resolution and volume 
control, and improves the overall process throughput. The high sensitivity to UV irradiation was related to a fast UV curing rate, which enables an ambient temperature imprint process and improves the overall throughput in mass fabrication. The low film volumetric shrinkage of the nanoimprint resist material in the UV curing reaction is necessary to maintain both patterning dimensional accuracy and lithographic resolution in UV curing nanoimprint lithography, in order to prevent pattern distortions and crack formations during the UV curing reactions. The nanoimprint resist material needs to have suitable mechanical strength and thermal stability, to survive the environmental stress during the nanoimprint and etch processes.

The chemical structures of the developed nanoimprint resist material containing a UV reactive fluorine surfactant are shown in Figure 3. Heptadecafluoro-2-hydroxyundecyl acrylate as a UV reactive fluorine surfactant was blended with tricylodecane dimethanol diacrylate as a bulk polymerizable organic monomer, dipentaerythritol polyacrylate-based composition as a UV crosslinker, and 2-hydroxy-2-methyl-1-phenylpropane-1-one as a photoinitiator. The bulk polymerizable organic monomers had low viscosity and can be rapidly polymerized. The UV crosslinker had the suitable mechanical strength and the thermal stability of the polymerized nanoimprint resist. The liquid photoinitiator needed to have a UV curing reaction. The nanoimprint resist materials are often formulated from the above process requirements with additional modifications tailored for the specific application. Table 1 shows the formulation of the prepared nanoimprint resist materials at three different weight ratios of heptadecafluoro-2-hydroxyundecyl acrylate; $9.0 \mathrm{wt} \%$ (sample name: TPULR9), $4.5 \mathrm{wt} \%$ (TPULR4.5), and $0 \mathrm{wt} \%$ (TPULR0). The resulting nanoimprint resist materials were filtered through an ultrahigh-molecular-weight polyethylene membrane filter having an average pore size of $50 \mathrm{~nm}$.

Figure 3. Chemical structures of the nanoimprint resist material containing a UV reactive fluorine surfactant: (a) heptadecafluoro-2-hydroxyundecyl acrylate; (b) tricylodecane dimethanol diacrylate; (c) dipentaerythritol polyacrylate-based composition; and (d) 2-hydroxy-2-methyl-1-phenylpropane-1-one.

(a)<smiles>C=CC(=O)OC[C@@H](O)CC(F)(F)C(F)(F)C(F)(F)C(F)(F)C(F)(F)C(F)(F)C(F)(F)C(F)(F)F</smiles>

(c)<smiles>C=CC(=O)COC(COCC(COC(=O)C=C)(COC(=O)C=C)COC(=O)C=C)(COC(=O)C=C)COC(=O)C=C</smiles>

(b)<smiles>C=C(C)OCCCC1CC2CCC1C1CCCC21</smiles>

(d)<smiles>CC(C)(O)C(=O)c1ccccc1</smiles> 
Table 1. Formulation of the developed nanoimprint resist materials TPULR9, TPULR4.5, and TPULR0.

\begin{tabular}{lcccc}
\hline $\begin{array}{l}\text { Resist } \\
\text { material }\end{array}$ & $\begin{array}{c}\text { Heptadecafluoro- } \\
\text { 2-hydroxyundecyl } \\
\text { acrylate }\end{array}$ & $\begin{array}{c}\text { Tricylodecane } \\
\text { dimethanol } \\
\text { diacrylate }\end{array}$ & $\begin{array}{c}\text { Di-pentaerythritol } \\
\text { polyacrylate }\end{array}$ & $\begin{array}{c}\text { 2-Hydroxy-2- } \\
\text { methyl-1-phenyl- } \\
\text { propane-1-one }\end{array}$ \\
\hline TPULR0 & $0 \mathrm{wt} \%$ & $47 \mathrm{wt} \%$ & $47 \mathrm{wt} \%$ & $6.0 \mathrm{wt} \%$ \\
TPULR4.5 & 4.5 & 45 & 45 & 5.5 \\
TPULR9 & 9 & 43 & 43 & 5 \\
\hline
\end{tabular}

The spin-on underlayer material with terminal methacrylate groups was developed to improve the dirty-template-causing resist pattern peeling and defect on the template due to stronger adhesion between the resist material and the underlayer, compared with that between the template and resist material [3]. The underlayer was then named as TPU-UL.

\subsection{Solvent Resistance of Nanoimprint Resist Materials}

In order to induce the anti-adhesion of the nanoimprint resist material to the template, the resistances of the above nanoimprint resist materials to the solvents were evaluated. In this procedure, the solutions of the above-mentioned solvent-diluted nanoimprint resist materials TPULR9, TPULR4.5, and TPULR0 were spun onto the prepared underlayer material TPU-UL at 2,500 rpm for $60 \mathrm{~s}$ and then UV-polymerized. UV polymerization was carried out by using a metal halide lamp system (Sun Energy, broad band targeted for the $365 \mathrm{~nm}$ peak, irradiation intensity: $58 \mathrm{~mW} / \mathrm{cm}^{2}$, irradiation time: $40 \mathrm{~s}$ ). The thicknesses of TPULR9, TPULR4.5, and TPULR0 were then measured using a thin film measurement system. Ethyl lactate was dispensed on the coated resist materials, and then kept for $120 \mathrm{~s}$, followed by spin drying at 5,000 rpm for $60 \mathrm{~s}$. The thickness of each resist layer was then re-measured. The amount of stripping was determined as the difference between the initial and final thicknesses of the resist layers.

\subsection{Surface Free Energy of Nanoimprint Resist Materials}

The surface free energy of nanoimprint resist materials is essential to prevent the generated resist based defects on the template when the template is removed from the nanoimprint resist material after pressing the template into the nanoimprint resist material. The surface free energies of TPULR9, TPULR4.5, and TPULR0 were calculated using the reported surface free energy components of water, n-hexadecane, and diiodomethane, together with the experimental contact angles of water, n-hexadecane, and diiodomethane on TPULR9, TPULR4.5, and TPULR0 layers. The following Equations (1), (2), and (3) were used, proposed by Owens [10] who extended the Fowkes concept [11].

$$
\begin{gathered}
\gamma_{L}(1+\cos \theta)=2\left(\gamma_{S}^{\mathrm{d}} \cdot \gamma_{\mathrm{L}}^{\mathrm{d}}\right)^{1 / 2}+2\left(\gamma_{\mathrm{S}}^{\mathrm{p}} \cdot \gamma_{\mathrm{L}}^{\mathrm{p}}\right)^{1 / 2}+2\left(\gamma_{\mathrm{S}}^{\mathrm{h}} \cdot \gamma_{\mathrm{L}}^{\mathrm{h}}\right)^{1 / 2} \\
\gamma_{\mathrm{S}}=\gamma_{\mathrm{S}}^{\mathrm{d}}+\gamma_{\mathrm{S}}^{\mathrm{p}}+\gamma_{\mathrm{S}}^{\mathrm{h}} \\
\gamma_{\mathrm{L}}=\gamma_{\mathrm{L}}^{\mathrm{d}}+\gamma_{\mathrm{L}}^{\mathrm{p}}+\gamma_{\mathrm{L}}^{\mathrm{h}}
\end{gathered}
$$


In the criterion, $\gamma_{\mathrm{L}}$ is surface free energy, $\gamma_{\mathrm{L}}^{\mathrm{d}}$ is the dispersive component, $\gamma_{\mathrm{L}}^{\mathrm{p}}$ is the polar component, and $\gamma_{\mathrm{L}}^{\mathrm{h}}$ is the hydrogen-bonded component of the liquid solvent. $\gamma_{\mathrm{S}}$ is surface free energy of the solid nanoimprint resist materials, $\gamma_{S}^{\mathrm{d}}$ is the dispersive component, $\gamma_{\mathrm{S}}^{\mathrm{p}}$ is the polar component, and $\gamma_{\mathrm{S}}^{\mathrm{h}}$ is the hydrogen-bonded component.

In the contact angle measurement process, which enables the determination of the surface free energy, three types of solvents, water, n-hexadecane, and diiodomethane, were selected, and the surface free energy components were reported as shown in Table 2 [12].

Table 2. Surface free energy components of water, n-hexadecane, and diiodomethane: $\gamma_{\mathrm{L}}$ surface free energy, $\gamma_{L}^{\mathrm{d}}$ dispersive component, $\gamma_{\mathrm{L}}^{\mathrm{p}}$ polar component, and $\gamma_{\mathrm{L}}^{\mathrm{h}}$ hydrogen-bonded component.

\begin{tabular}{lcccr}
\hline Liquid & $\boldsymbol{\gamma}_{\mathbf{L}}$ & $\boldsymbol{\gamma}_{\mathbf{L}}^{\mathbf{d}}$ & $\boldsymbol{\gamma}_{\mathbf{L}}^{\mathbf{p}}$ & $\boldsymbol{\gamma}_{\mathbf{L}}^{\mathbf{h}}$ \\
\hline Water & 72.8 & 29.1 & 1.3 & 42.4 \\
n-Hexadecane & 27.6 & 27.6 & 0.0 & 0.0 \\
Diiodomethane & 50.8 & 46.8 & 4.0 & 0.0 \\
\hline
\end{tabular}

TPULR9, TPULR4.5, and TPULR0 were spin-coated onto the underlayer material TPU-UL at $2,500 \mathrm{rpm}$ for $60 \mathrm{~s}$ and then UV-polymerized. The contact angles of water, $\mathrm{n}$-hexadecane, and diiodomethane on TPULR9, TPULR4.5, and TPULR0 layers were measured using a dynamic contact angle analyzer DropMaster500Z (Kyowa Interface Science) as follows (analysis method: $\theta / 2$ method, drop amount: $1.0 \mu \mathrm{L}$, measurement time after droping: $1.0 \mathrm{~s}$ ).

\subsection{Film Volumetric Shrinkage During UV Curing Process}

Minimal film volumetric shrinkage during the UV curing process is one of the important criteria for developing the nanoimprint resist material. The amount of volumetric shrinkage was determined as the difference between the initial and final thicknesses in the UV curing process. TPULR9, TPULR4.5, and TPULR0 were spun onto the prepared underlayer material TPU-UL at 2,500 rpm for $60 \mathrm{~s}$ and then baked on a hotplate at $90{ }^{\circ} \mathrm{C}$ for $30 \mathrm{~s}$. The thickness was then measured. UV polymerization was carried out by using a metal halide lamp system (Sun Energy, broad band targeted for the $365 \mathrm{~nm}$ peak, irradiation intensity: $58 \mathrm{~mW} / \mathrm{cm}^{2}$, irradiation time: $40 \mathrm{~s}$ ). The thickness was re-measured after the UV curing process.

\section{Results and Discussion}

\subsection{Solvent Resistance of Nanoimprint Resist Materials}

TPULR9, TPULR4.5, and TPULR0 must have the UV polymerizable property and solvent resistance in order to achieve the excellent patterning dimensional accuracy. The evaluation of solvent resistance using ethyl lactate showed no detectable changes in film thickness, indicating that TPULR9, TPULR4.5, and TPULR0 do not significantly interact. It was obvious that TPULR9, TPULR4.5, and TPULR0 were polymerized on the basis of their solvent resistance. 


\subsection{Effect of Surface Free Energy of Nanoimprint Resist Materials Containing a UV Reactive Fluorine Surfactant on Defect Reduction in Nanoimprint Patterning Replication}

Table 3 shows the calculated surface free energy components of TPULR9, TPULR4.5, and TPULR0 with different concentrations of UV reactive fluorine surfactant.

Table 3. Surface free energy components of TPULR9, TPULR4.5, and TPULR0.

\begin{tabular}{lcccc}
\hline Resist material & $\boldsymbol{\gamma}_{\mathbf{S}}$ & $\boldsymbol{\gamma}_{\mathbf{S}}^{\mathbf{d}}$ & $\boldsymbol{\gamma}_{\mathbf{S}}^{\mathbf{p}}$ & $\boldsymbol{\gamma}_{\mathbf{S}}^{\mathbf{h}}$ \\
\hline TPULR0 & 47 & 27 & 12 & 8.1 \\
TPULR4.5 & 20 & 16 & 3.6 & 0.1 \\
TPULR9 & 22 & 21 & 0.5 & 0.0 \\
\hline
\end{tabular}

The surface free energy of TPULR9, TPULR4.5, and TPULR0 were determined as 22, 20 and 47, respectively. TPULR4.5 and TPULR9 containing 4,4,5,5,6,6,7,7,8,8,9,9,10,10,11,11, 11-heptadecafluoro-2-hydroxyundecyl acrylate showed a better range of surface free energy than TPULR0. This was because the surface free energy in the nanoimprint resist materials was considered to be related to the rounding of sharp corners due to the surface free energy after the template was released from the surface of the nanoimprinted resist material on the template. Therefore, the reduction of the surface free energy in TPULR4.5 and TPULR9 was expected to lead to stronger anti-adhesion. The surface free energy and the components were related to the concentration of $4,4,5,5,6,6,7,7,8,8,9,9,10,10,11,11,11$-heptadecafluoro-2-hydroxyundecyl acrylate as shown in Table 1 .

The surface free energy and the components decreased generally as the concentration of the prepared UV reactive fluorine surfactant in the resist material increased. In the material combination of the developed resist TPULR4.5 and TPULR9 on underlayer TPU-UL, it was not possible to find the significant difference of surface energy value between $4.5 \mathrm{wt} \%$ and $9 \mathrm{wt} \%$, as the concentration of the UV reactive fluorine surfactant increases more than $4.5 \mathrm{wt} \%$. In order to investigate the cause of the observed relationship between surface energy value and materials, the influences such as UV cross-link reaction ratio, the surface energy value of the underlayer, phase separation, and micro-morphology were considered. The optimized concentration was found to be $4.5 \mathrm{wt} \%$, in order to develop the new nanoimprint resist with great repeatability and durability of the nanoimprint processes. Therefore, TPULR4.5 was used in this study being acceptable for the evaluation of repeatability and durability of nanoimprint processes.

\subsection{UV Curing Nanoimprint Lithography}

The repeatable properties of producing lines and trenches with a $500 \mathrm{~nm}$ contact hole and $2 \mu \mathrm{m}$ line patterns on the surface of TPU-UL were evaluated, to observe the effect of reducing the defect numbers on the template in the replication of nanoimprint process cycles. The same template was used to imprint TPULR4.5 and TPULR0 over 20 times. Figure 4 shows the optical microscope images of the template with a $500 \mathrm{~nm}$ contact hole and $2 \mu \mathrm{m}$ line patterns in TPULR4.5 containing $4,4,5,5,6,6,7,7,8,8,9,9,10,10,11,11,11$-heptadecafluoro-2-hydroxyundecyl acrylate as a UV reactive fluorine surfactant and TPULR0. 
Figure 4. Optical microscope images of (a) template and (b) nanoimprint resist material on underlayer with $500 \mathrm{~nm}$ contact hole and $2 \mu \mathrm{m}$ line patterns.

\begin{tabular}{|c|c|c|c|c|}
\hline \multirow{2}{*}{ Sample name } & \multicolumn{4}{|c|}{ Number of nanoimprint process cycles } \\
\hline & Before test & 1 & 10 & 20 \\
\hline \multicolumn{5}{|l|}{ TPULRO } \\
\hline \multicolumn{5}{|l|}{ TPULR4.5 } \\
\hline & $\overleftarrow{50 \mu n}$ & & & \\
\hline
\end{tabular}

(a)

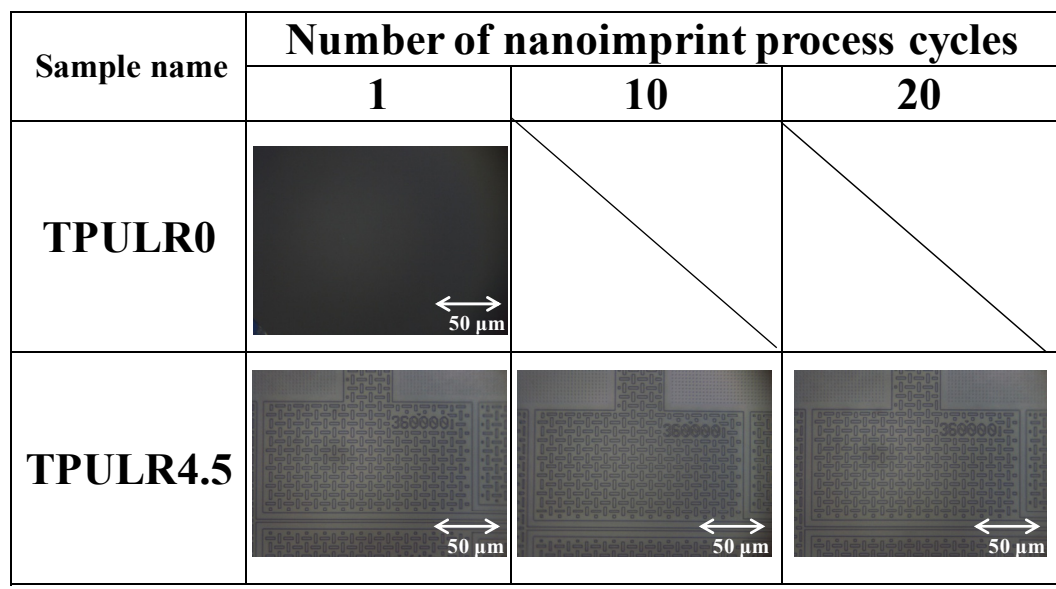

(b)

The resist patterns of the referenced TPULR0 without a UV reactive fluorine surfactant were non-imprinted after one imprint and almost part of the resist patterns was observed on the template. The template separation failure was considered to be due to the fact that the adhesion between TPULR 0 and template was stronger compared with that between TPULR 0 and the underlayer material TPU-UL, in the case of no approach to use a coated fluorinated self assembled monolayer on the surface of template.

On the other hand, no noticeable changes on the template with a non-coated fluorinated self assembled monolayer after 20 nanoimprint process cycles were achieved by using the developed TPULR4.5 on TPU-UL. TPULR4.5 on TPU-UL indicated excellent patterning dimensional accuracy in the replication of 20 nanoimprint process cycles. Anti-adhesion was found between the template and TPULR4.5 containing a UV reactive fluorine surfactant. The nanoimprint lithography over 20 nanoimprint process cycle times can be expected to give good repeatability and durability. The surfactant 4,4,5,5,6,6,7,7,8,8,9,9,10,10,11,11,11-heptadecafluoro-2-hydroxyundecyl acrylate as a template release component included in TPULR4.5 was confirmed to be effective for the reduction of contamination and defect of nanoimprint resist material on the template,and thus as a clean separation technology for mass-production using UV curing nanoimprint lithography. It was also believed that 
this approach to use the developed nanoimprint resist materials containing UV reactive fluorine surfactants, produced high fidelity replication by defect reduction, with a single polymeric material as a disposable nanoimprint template instead of quartz or silica templates.

The common surface modification technique in nanoimprint lithography applied a fluorinated self assembled monolayer on the template. The fluorinated self assembled monolayer reacts with the free silanol groups on the surface of the quartz template in a condensation reaction to form a single layer of fluorinated alkyl chains. The treatment using a fluorinated self assembled monolayer is found to degrade after repeated nanoimprints, suggesting a breakdown or erosion of the fluorinated monolayer $[13,14]$. The used template surface in this study was not treated with a common fluorinated self assembled monolayer. Future study to reduce the defects generated from de-molding is expected; in addition to use of a common fluorinated self assembled monolayer on the template.

\subsection{Film Volumetric Shrinkage during UV Curing Process}

The experimental film volumetric shrinkages during the UV curing process in TPULR9, TPULR4.5, and TPULR0 were 9.2, 9.0 and 8.9\%, respectively. The film volumetric shrinkages of the different nanoimprint resist materials have been investigated in the previous study [13]. The film volumetric shrinkages of mr-UVCur06 (Micro Resist Technology) with a liquid UV curing polymer system of low viscosity and high curing rate as a commercial nanoimprint material, and a second acrylate type resist consisting of n-butyl acrylate (27 wt\%), isobornyl acrylate (50 wt\%), ethylene glycol diacrylate (20 wt \%), and 2-hydroxy-2-methyl-1-phenyl-1-propanone (3 wt \%) were 8.3 and $9.0 \%$, respectively.

It was found that TPULR9, TPULR4.5, and TPULR0 had good shrinkage of film thickness, compared with the referenced resist materials. The bulky structure in tricylodecane dimethanol diacrylate was considered to be effective for minimizing film shrinkage.

\section{Conclusions}

The developed nanoimprint resist material containing a UV reactive fluorine surfactant as a template release component was successfully applied in UV curing nanoimprint lithography. The development of the nanoimprint resist material and the associated lithography enabled the patterning of a $500 \mathrm{~nm}$ contact hole and $2 \mu \mathrm{m}$ lines on the underlayer over 20 nanoimprint process cycles. The optimized formulation and the surface free energy components of the nanoimprint resist material were considered to lead to the high anti-adhesion between template and the nanoimprint resist material. It was also shown that the nanoimprint process cycles could be performed using the same template, without the use of a fluorinated self assembled monolayer on the template as a common surface modification technique.

\section{Acknowledgments}

The authors are deeply grateful to Electronic Material Research Laboratories of Nissan Chemical Industries, Ltd and C. Grant Willson of the University of Texas at Austin for technical advice in UV curing nanoimprint lithography. This work was funded by a Konica Minolta Science and Technology Foundation. 


\section{References}

1. Willson, C.G. A decade of step and flash imprint lithography. J. Photopolym. Sci. Technol. 2009, $22,147-153$.

2. Takei, S. Development of ultraviolet crosslinking glucose-based resist materials for advanced electronic device applications using nanoimprint lithography. Jpn. J. Appl. Phys. 2011, 50, 01BA02:1-01BA02:4.

3. Takei, S.; Ogawa, T.; Deschner, R.; Hanabata, M.; Willson, C.G. Advanced step and flash nano imprint lithography using ultraviolet sensitive hard mask underlayer material. Micro Nano Lett. 2010, 5, 117-120.

4. Takei, S. Ultraviolet nano imprint lithography using fluorinated silicon-based resist materials. Appl. Phys. Express 2010, 3, 025203:1-025203:3.

5. Nagase, K.; Kubo, S.; Nakagawa, M. Resist properties of thin poly(methyl methacrylate) and polystyrene films patterned by thermal nanoimprint lithography for Au electrodeposition. Jpn. J. Appl. Phys. 2010, 49, 075201:1-075201:5.

6. Youn, S.W.; Hiroshima, H.; Takahashi, M.; Maeda, R. Size dependence of quick cavity filling behavior in ultraviolet nanoimprint lithography using pentafluoropropane gas. Jpn. J. Appl. Phys. 2010, 49, 06GL06:1-06GL06:4.

7. Okada, M.; Nakayama, T.; Kang, Y.; Haruyama, Y.; Kanda, K.; Matsui, S. Direct patterning on sol-gel low-k porous silica by thermal nanoimprinting. Jpn. J. Appl. Phys. 2010, 49, 06GL08:1-06GL08:3.

8. Singh, L.; Luo, K.; Ye, Z.; Xu, F. Haase, G.; Curran, D.; LaBrake, D.; Resnick, D.; Sreenivasan, S.V. Defect reduction of high-density full-field patterns in jet and flash imprint lithography. $J$. Micro/Nanolithogr. MEMS MOEMS 2011, 10, doi:10.1117/1.3625635.

9. Takei, S.; Ogawa, T.; Willson, C.G. Study of fluorinated silicon-based resist material and photoreactive underlayer for defect reduction in step and flash nanoimprint lithography. Micro Nano Lett. 2011, 6, 442-424.

10. Owens, D.K.; Wendt, R.C. Estimation of the surface free energy of polymers. J. Appl. Polym. Sci. 1969, 13, 1741-1747.

11. Fowkes, F.M. Additivity of intermolecular forces at interfaces. I. determination of the contribution to surface and interfacial tensions of dispersion forces in various liquids. J. Phys. Chem. 1963, 67, $2538-2541$.

12. Shouro, D.; Ito, K.; Takata, T. Study on molecular structure in the surface of polyethylene terephthalate fiber for the industrial use. Sen'i Gakkaishi 2005, 61, 177-182.

13. Takei, S. Step and flash nano imprint lithography of $80 \mathrm{~nm}$ dense line pattern using trehalose derivative resist material. Appl. Phys. Express 2010, 3, 025202:1-025202:3.

14. Wang, Q.; Hiroshima, H. Effects of environmental gas in UV nanoimprint on the characteristics of UV-curable resin. Jpn. J. Appl. Phys. 2010, 49, 06GL04:1-06GL04:5.

(C) 2012 by the authors; licensee MDPI, Basel, Switzerland. This article is an open access article distributed under the terms and conditions of the Creative Commons Attribution license (http://creativecommons.org/licenses/by/3.0/). 